\title{
RADIAL FUNCTIONS AND MAXIMAL ESTIMATES FOR SOLUTIONS TO THE SCHRÖDINGER EQUATION
}

\author{
PER SJÖLIN
}

(Received 20 September 1992; revised 29 January 1993)

Communicated by A. H. Dooley

\begin{abstract}
Maximal estimates are considered for solutions to an initial value problem for the Schrödinger equation. The initial value function is assumed to be radial in $\mathbb{R}^{n}, n \geq 2$.

1991 Mathematics subject classification (Amer. Math. Soc.): 42B25, 35Q40.
\end{abstract}

Let $f$ belong to the Schwartz space $\mathscr{S}\left(\mathbb{R}^{n}\right)$ and set

$$
S_{t} f(x)=u(x, t)=(2 \pi)^{-n} \int_{\mathbb{R}^{n}} e^{i x \cdot \xi} e^{i t|\xi|^{n}} \widehat{f}(\xi) d \xi, \quad x \in \mathbb{R}^{n}, t \in \mathbb{R},
$$

where $a>1$. Here $\widehat{f}$ denotes the Fourier transform of $f$, defined by

$$
\widehat{f}(\xi)=\int_{\mathbb{R}^{n}} e^{-i \xi \cdot x} f(x) d x .
$$

We then have $u(x, 0)=f(x)$, and in the case $a=2, u$ is a solution to the Schrödinger equation $\Delta u=i \partial u / \partial t$. We set

$$
S^{*} f(x)=\sup _{0<t<1}\left|S_{t} f(x)\right|, \quad x \in \mathbb{R}^{n} .
$$

We also introduce Sobolev spaces $H_{s}$ by setting

$$
H_{s}=\left\{f \in \mathscr{S}^{\prime} ;\|f\|_{H_{s}}<\infty\right\}, \quad s \in \mathbb{R},
$$

where

$$
\|f\|_{H_{s}}=\left(\int_{\mathbb{R}^{n}}\left(1+|\xi|^{2}\right)^{s}|\widehat{f}(\xi)|^{2} d \xi\right)^{1 / 2}
$$

This research was supported by the Swedish Natural Science Research Council.

(C) 1995 Australian Mathematical Society 0263-6115/95 \$A2.00+0.00 
We shall here study estimates of the type

$$
\left(\int_{B(0 ; R)}\left|S^{*} f(x)\right|^{2} d x\right)^{1 / 2} \leq C_{R}\|f\|_{H_{s}}, f \in \mathscr{S}\left(\mathbb{R}^{n}\right),
$$

where $B(0 ; R)=\left\{x \in \mathbb{R}^{n} ;|x| \leq R\right\}$. The inequality (1) has implications for the existence almost everywhere of $\lim _{t \rightarrow 0} u(x, t)$ for solutions $u$ of the Schrödinger equation. These problems were first studied by Carleson [3]. Later the inequality (1) and related questions were studied in several papers: see for example Dahlberg and Kenig [5], Kenig and Ruiz [6], Carbery [2], Cowling [4], Sjölin [10], Vega [12] and Kenig, Ponce and Vega [7]. The following results are known. For $n=1,(1)$ holds with $s=1 / 4$, and $1 / 4$ cannot be replaced by a smaller number. In one variable one also has the improvement

$$
\left(\int_{B(0 ; R)}\left|S^{*} f(x)\right|^{4} d x\right)^{1 / 4} \leq C_{R}\|f\|_{H_{1 / 4}} .
$$

For $n=2$, (1) holds with $s=1 / 2$ and in the case $n=2, a=2$, Bourgain [1] also has a result for $H_{s}\left(\mathbb{R}^{2}\right)$ for some $s<1 / 2$. In the case $n \geq 3$, (1) is known to hold for $s>1 / 2$.

For radial functions in $\mathbb{R}^{n}, n \geq 2$, Prestini [9] has proved that

$$
\int_{B(0 ; R)} S^{*} f(x) d x \leq C_{R}\|f\|_{H_{1 / 4}}
$$

and here $1 / 4$ cannot be replaced by a smaller number.

The purpose of this paper is to improve the integrability in the left hand side of (3). For $n \geq 2$ we shall prove the following results.

THEOREM 1. If $q=4 n /(2 n-1)$, then for $f$ radial,

$$
\left(\int_{B(0 ; R)}\left|S^{*} f(x)\right|^{q} d x\right)^{1 / q} \leq C_{R}\|f\|_{H_{1 / 4}} .
$$

If $q>4 n /(2 n-1)$, then the estimate (4) does not hold for all radial functions $f$. Theorem 1 is a direct consequence of the following theorem.

THEOREM 2. Assume $2 \leq q \leq 4$. If $\alpha=q(2 n-1) / 4-n$ and $f$ is radial, then

$$
\left(\int_{B(0 ; R)}\left|S^{*} f(x)\right|^{q}|x|^{\alpha} d x\right)^{1 / q} \leq C_{R}\|f\|_{H_{1 / 4}} .
$$

If $\alpha<q(2 n-1) / 4-n$ then (5) does not hold for all radial functions $f$. 
In the proof of Theorem 2 we shall use Pitt's inequality for Fourier transforms, which states that

$$
\left(\int_{\mathbb{R}}|\widehat{f}(\xi)|^{q}|\xi|^{-\gamma q} d \xi\right)^{1 / q} \leq C\left(\int_{\mathbb{R}}|f(x)|^{p}|x|^{\alpha p} d x\right)^{1 / p},
$$

if $q \geq p, 0 \leq \alpha<1-1 / p, 0 \leq \gamma<1 / q$ and $\gamma=\alpha+1 / p+1 / q-1$ (see for instance Muckenhoupt [8]). We take $q=2$ and $\gamma=1 / 4$ in (6) and then obtain

$$
\left(\int_{\mathbb{R}}|\widehat{f}(\xi)|^{2}|\xi|^{-1 / 2} d \xi\right)^{1 / 2} \leq C\left(\int_{\mathbb{R}}|f(x)|^{p}|x|^{3 p / 4-1} d x\right)^{1 / p}
$$

for $4 / 3 \leq p \leq 2$.

ProOF OF THEOREM 2. We assume $2 \leq q \leq 4$ and $1 / p+1 / q=1$ so that $4 / 3 \leq p \leq 2$. We let $t(x)$ be a measurable and radial function in $\mathbb{R}^{n}$ with $0<t(x)<1$ and set $T f(x)=S_{t(x)} f(x), f \in \mathscr{S}$. It is then sufficient to prove (5) with $S^{*}$ replaced by $T$.

If $f$ is radial we obtain $S_{t} f(s)=c_{n} s^{1-n / 2} \int_{0}^{\infty} J_{n / 2-1}(r s) e^{i t r^{a}} \widehat{f}(r) r^{n / 2} d r$, where $J_{n / 2-1}$ denotes a Bessel function (see Stein and Weiss [11, p. 155]). Here we write $S_{t} f(s)=S_{t} f(x)$ if $s=|x|$ and $\widehat{f}(r)=\widehat{f}(\xi)$ if $r=|\xi|$.

Similarly, we obtain

$$
T f(s)=c_{n} s^{1-n / 2} \int_{0}^{\infty} J_{n / 2-1}(r s) e^{i t(s) r^{a}} \widehat{f}(r) r^{n / 2} d r .
$$

To prove (5) we have to prove that

$$
\left(\int_{0}^{R}|T f(s)|^{q} s^{q(2 n-1) / 4-1} d s\right)^{1 / q} \leq C_{R}\left(\int_{0}^{\infty}|\widehat{f}(r)|^{2}\left(1+r^{2}\right)^{1 / 4} r^{n-1} d r\right)^{1 / 2} .
$$

We have

$$
\begin{aligned}
T f(s) s^{(2 n-1) / 4-1 / q} & =c_{n} s^{(2 n-1) / 4-1 / q+1-n / 2} \int_{0}^{\infty} J_{n / 2-1}(r s) e^{i t(s) r^{a}} \widehat{f}(r) r^{n / 2} d r \\
& =c_{n} s^{3 / 4-1 / q} \int_{0}^{\infty} J_{n / 2-1}(r s) e^{i t(s) r^{a}} g(r)\left(1+r^{2}\right)^{-1 / 8} r^{1 / 2} d r,
\end{aligned}
$$

where $g(r)=\widehat{f}(r)\left(1+r^{2}\right)^{1 / 8} r^{(n-1) / 2}$. We set

$$
P g(s)=s^{3 / 4-1 / q} \int_{0}^{\infty} J_{n / 2-1}(r s) e^{i t(s) r^{a}} g(r)\left(1+r^{2}\right)^{-1 / 8} r^{1 / 2} d r
$$

and then have

$$
T f(s) s^{(2 n-1) / 4-1 / q}=c_{n} P g(s) .
$$


We have to prove that

$$
\left(\int_{0}^{R}|P g(s)|^{q} d s\right)^{1 / q} \leq C_{R}\left(\int_{0}^{\infty}|g(r)|^{2} d r\right)^{1 / 2} .
$$

The basic idea in the proof of (9) is to estimate the adjoint of $P$ by use of an inequality in our paper [10]. We set

$$
P^{*} g(r)=\left(1+r^{2}\right)^{-1 / 8} r^{1 / 2} \int_{0}^{R} J_{n / 2-1}(r s) e^{-i t(s) r^{a}} s^{3 / 4-1 / q} g(s) d s, \quad 0<r<\infty,
$$

if $g \in L^{1}(0, R)$. It is then easy to prove that

$$
\int_{0}^{\infty} f(r) \overline{P^{*} g(r)} d r=\int_{0}^{R} P f(s) \overline{g(s)} d s
$$

if $g \in L^{1}(0, R), f \in L^{2}(0, \infty)$ and $f$ has a suitable decay at infinity. It is therefore sufficient to prove that

$$
\left(\int_{0}^{\infty}\left|P^{*} g(r)\right|^{2} d r\right)^{1 / 2} \leq C_{R}\left(\int_{0}^{R}|g(s)|^{p} d s\right)^{1 / p}, \quad g \in L^{p}(0, R),
$$

for $4 / 3 \leq p \leq 2$.

It is well-known that there exist constants $b_{1}$ and $b_{2}$ such that

$$
\left|J_{n / 2-1}(t)-\left(b_{1} e^{i t} / t^{1 / 2}+b_{2} e^{-i t} / t^{1 / 2}\right)\right| \leq C / t^{3 / 2}, \quad t>1,
$$

(see $[11$, p. 158]) and we therefore have

$$
\left|t^{1 / 2} J_{n / 2-1}(t)-\left(b_{1} e^{i t}+b_{2} e^{-i t}\right)\right| \leq C / t, \quad t>1 .
$$

It is also clear that

$$
\left|t^{1 / 2} J_{n / 2-1}(t)-\left(b_{1} e^{i t}+b_{2} e^{-i t}\right)\right| \leq C, \quad 0<t \leq 1 .
$$

Setting $\gamma=1 / q-1 / 4$ we have $s^{3 / 4-1 / q}=s^{1 / 2} s^{-\gamma}$ and it follows that

$$
\begin{aligned}
P^{*} g(r)= & b_{1}\left(1+r^{2}\right)^{-1 / 8} \int_{0}^{R} e^{i r s} e^{-i t(s) r^{a}} s^{-\gamma} g(s) d s \\
& +b_{2}\left(1+r^{2}\right)^{-1 / 8} \int_{0}^{R} e^{-i r s} e^{-i t(s) r^{a}} s^{-\gamma} g(s) d s+Q(r) \\
= & b_{1} A(r)+b_{2} B(r)+Q(r),
\end{aligned}
$$

where

$$
|Q(r)| \leq C\left(1+r^{2}\right)^{-1 / 8} \int_{0}^{R} \min (1,1 / r s) s^{-\gamma}|g(s)| d s .
$$


We extend $A$ to $\mathbb{R}$ by setting

$$
A(\xi)=\left(1+\xi^{2}\right)^{-1 / 8} \int_{0}^{R} e^{i\left(\xi s-t(s)|\xi|^{a}\right)} s^{-\gamma} g(s) d s, \quad-\infty<\xi<0 .
$$

Then $B(\xi)=A(-\xi), 0<\xi<\infty$, and to estimate $A$ and $B$ it is therefore sufficient to prove that

$$
\left(\int_{-\infty}^{\infty}|A(\xi)|^{2} d \xi\right)^{1 / 2} \leq C_{R}\|g\|_{p}
$$

where

$$
\mid g \|_{p}=\left(\int_{0}^{R}|g(s)|^{p} d s\right)^{1 / p} .
$$

Choose $\rho$ real-valued in $C_{0}^{\infty}(\mathbb{R})$ such that $\rho(\xi)=1,|\xi| \leq 1$, and $\rho(\xi)=0,|\xi| \geq$ 2 , and set $\rho_{N}(\xi)=\rho(\xi / N)$ for $N>1$. Then set

$$
A_{N}(\xi)=\rho_{N}(\xi)|\xi|^{-1 / 4} \int_{0}^{R} e^{i\left(s \xi-t(s)|\xi|^{a}\right)} s^{-\gamma} g(s) d s
$$

We shall prove that

$$
\left(\int_{\mathbb{R}}\left|A_{N}(\xi)\right|^{2} d \xi\right)^{1 / 2} \leq C_{R}\|g\|_{p}
$$

with $C_{R}$ independent of $N$, and (12) follows from this inequality.

We have

$$
\begin{aligned}
\int_{\mathbb{R}} \mid & \left.A_{N}(\xi)\right|^{2} d \xi=\int_{\mathbb{R}} A_{N}(\xi) \overline{A_{N}(\xi)} d \xi \\
& =\int_{\mathbb{R}} \rho_{N}(\xi)^{2}|\xi|^{-1 / 2}\left(\int_{0}^{R} e^{i\left(s \xi-t(s)|\xi|^{\alpha}\right)} s^{-\gamma} g(s) d s\right) \cdot\left(\int_{0}^{R} e^{-i\left(s^{\prime} \xi-t\left(s^{\prime}\right)|\xi|^{\alpha}\right)} s^{\prime \gamma} \overline{g\left(s^{\prime}\right)} d s^{\prime}\right) d \xi \\
& =\int_{0}^{R} \int_{0}^{R}\left(\int_{\mathbb{R}} e^{i\left[\left(s-s^{\prime}\right) \xi-\left(t(s)-t\left(s^{\prime}\right)\right)|\xi|^{a}\right]} \rho_{N}(\xi)^{2}|\xi|^{-1 / 2} d \xi\right) s^{-\gamma} g(s) s^{\prime-\gamma} \overline{g\left(s^{\prime}\right)} d s d s^{\prime} .
\end{aligned}
$$

It is proved in [10, pp. 709-712], that the inner integral is bounded by $C\left|s-s^{\prime}\right|^{-1 / 2}$ and we therefore obtain

$$
\left|A_{N} \|_{2}^{2} \leq C \int_{\mathbb{R}} \int_{\mathbb{R}}\right| s-\left.s^{\prime}\right|^{-1 / 2} s^{-\gamma}|g(s)| s^{\prime-\gamma}\left|g\left(s^{\prime}\right)\right| d s d s^{\prime},
$$

where we have extended $g$ to $\mathbb{R}$ by setting $g(s)=0$ outside $[0, R]$.

We shall now use the Riesz potential operator $I_{\beta}, 0<\beta<1$, defined by

$$
I_{\beta} f(x)=c_{\beta} \int_{\mathbb{R}}|x-y|^{-1+\beta} f(y) d y, \quad x \in \mathbb{R} .
$$

Here $c_{\beta}$ is chosen so that $\left(I_{\beta} f\right) \widehat{(\xi)}=|\xi|^{-\beta} \widehat{f}(\xi)$. 
Using Fourier transforms one then has

$$
\begin{aligned}
\mid A_{N} \|_{2}^{2} & \leq C \int_{\mathbb{R}} I_{1 / 2}\left(t^{-\gamma}|g|\right)(s) s^{-\gamma}|g(s)| d s \\
& =C \int_{\mathbb{R}}|\xi|^{-1 / 2}\left(s^{-\gamma}|g|\right) \widehat{(\xi)} \overline{\left(s^{-\gamma}|g|\right)(\xi)} d \xi \\
& =C \int_{\mathbb{R}}|\xi|^{-1 / 2}\left|\left(s^{-\gamma}|g|\right)-(\xi)\right|^{2} d \xi .
\end{aligned}
$$

This formula is justified since we may assume that $g$ is bounded and vanishes close to the origin.

Invoking (7) one then obtains

$$
\left\|A_{N}\right\|_{2} \leq C\left(\int_{\mathbb{R}}\left|s^{-\gamma} g\right|^{p}|s|^{3 p / 4-1} d s\right)^{1 / p}=C\|g\| p,
$$

since

$$
-\gamma p+\frac{3 p}{4}-1=-\left(\frac{1}{q}-\frac{1}{4}\right) p+\frac{3 p}{4}-1=-\frac{p}{q}+p-1=0 .
$$

It remains to prove that if $Q(r)$ satisfies (11), then

$$
\left(\int_{0}^{\infty}|Q(r)|^{2} d r\right)^{1 / 2} \leq C_{R}\|g\|_{p}
$$

For $0<r<1$ one has

$$
|Q(r)| \leq \int_{0}^{R} s^{-\gamma}|g| d s \leq\left(\int_{0}^{R} s^{-\gamma q} d s\right)^{1 / q} \mid g\left\|_{p} \leq C_{R}\right\| g \|_{p},
$$

since $\gamma q=1-q / 4<1$. Hence

$$
\left(\int_{0}^{1}|Q(r)|^{2} d r\right)^{1 / 2} \leq C_{R}\|g\|_{p}
$$

For $r>1$ it follows from (11) that

$$
|Q(r)| \leq C Q_{1}(r)+C Q_{2}(r)
$$

where $\quad Q_{1}(r)=r^{-1 / 4} \int_{0}^{1 / r} s^{-\gamma}|g| d s \quad$ and $\quad Q_{2}(r)=r^{-5 / 4} \int_{1 / r}^{R} s^{-1-\gamma}|g| d s$ (here we assume $R>1$ ).

Using a change of variable we obtain

$$
\int_{1}^{\infty} Q_{1}(r)^{2} d r=\int_{0}^{1} M_{1}(t)^{2} d t
$$


where

$$
\begin{aligned}
M_{1}(t) & =\frac{1}{t} Q_{1}\left(\frac{1}{t}\right)=\frac{1}{t} t^{1 / 4} \int_{0}^{t} s^{-\gamma}|g| d s \\
& =t^{-3 / 4} \int_{0}^{t} s^{-\gamma}|g| d s \leq \int_{0}^{t}(t-s)^{-3 / 4} s^{-\gamma}|g| d s \\
& \leq C I_{1 / 4}\left(s^{-\gamma}|g|\right)(t) .
\end{aligned}
$$

One has

$$
\left(I_{1 / 4}\left(s^{-\gamma}|g|\right)\right) \widehat{\mid}|\xi|=|\xi|^{-1 / 4}\left(s^{-\gamma}|g|\right) \widehat{(\xi)}
$$

and invoking Plancherel's theorem and arguing as above we obtain

$$
\int_{1}^{\infty} Q_{1}(r)^{2} d r \leq C \int_{\mathbb{R}}|\xi|^{-1 / 2}\left|\left(s^{-\gamma}|g|\right) \widehat{(\xi)}\right|^{2} d \xi \leq C\|g\|_{p}^{2} .
$$

It remains to estimate $Q_{2}(r)$. We have

$$
\int_{1}^{\infty} Q_{2}(r)^{2} d r=\int_{0}^{1} M_{2}(t)^{2} d t
$$

where

$$
\begin{aligned}
M_{2}(t) & =\frac{1}{t} Q_{2}\left(\frac{1}{t}\right)=t^{1 / 4} \int_{t}^{R} s^{-1-\gamma}|g| d s \leq \int_{t}^{R} s^{-3 / 4} s^{-\gamma}|g| d s \\
& \leq \int_{t}^{R}(s-t)^{-3 / 4} s^{-\gamma}|g| d s \leq C I_{1 / 4}\left(s^{-\gamma}|g|\right)(t),
\end{aligned}
$$

and it follows as above that

$$
\left(\int_{1}^{\infty} Q_{2}(r)^{2} d r\right)^{1 / 2} \leq C\|g\|_{p} .
$$

Hence (15) is proved and the proof of (5) is complete.

We shall now prove that (5) does not hold if $\alpha<q(2 n-1) / 4-n$. Therefore assume that (5) holds for $\alpha=q(2 n-1) / 4-n-\varepsilon$, where $\varepsilon>0$ is a small number. We shall prove that this leads to a contradiction.

Let $\varphi \in C_{0}^{\infty}\left(\mathbb{R}^{n}\right)$ be radial and non-negative. Assume that $\operatorname{supp} \varphi \subset\{\xi: 1<$ $|\xi|<2\}$ and that $\varphi(\xi)=1$ for $5 / 4 \leq|\xi| \leq 7 / 4$. Then set $\varphi_{c}(\xi)=\varphi(\xi / c), c>1$, and choose $f$ such that $\widehat{f}=\varphi_{c}$. It is then easy to see that

$$
\|f\|_{H_{1 / 4}} \leq C c^{n / 2+1 / 4}
$$

We have

$$
\begin{aligned}
S_{t} f(x) & =c_{n} \int_{\mathbb{R}^{n}} e^{i x \cdot \xi} e^{i t|\xi|^{a}} \varphi(\xi / c) d \xi \\
& =c_{n} \int_{\mathbb{R}^{n}} e^{i c x \cdot \eta} e^{i t|c \eta|^{a}} \varphi(\eta) d \eta c^{n}
\end{aligned}
$$


and

$$
S_{0} f(x)=c_{n} c^{n} \int_{\mathbb{R}^{n}} e^{i c x \cdot \eta} \varphi(\eta) d \eta=c_{n} c^{n} \widehat{\varphi}(c x)
$$

It follows that

$$
S^{*} f(x) \geq\left|S_{0} f(x)\right| \geq c_{0} c^{n}
$$

for $|x| \leq \delta / c$, where $c_{0}$ and $\delta$ are positive constants. For $R>\delta$ we therefore obtain

$$
\begin{aligned}
& \left(\int_{B(0 ; R)}\left|S^{*} f(x)\right|^{q}|x|^{\alpha} d x\right)^{1 / q} \geq c_{0}\left(\int_{|x| \leq \delta / c} c^{n q}|x|^{\alpha} d x\right)^{1 / q} \\
& \quad=c_{0} c^{n}\left(\int_{0}^{\delta / c} t^{\alpha+n-1} d t\right)^{1 / q} \geq c_{0} c^{n}\left(c^{-\alpha-n}\right)^{1 / q} \\
& \quad=c_{0} c^{n-(\alpha+n) / q}
\end{aligned}
$$

Now

$$
n-\frac{\alpha+n}{q}=n-\frac{2 n-1}{4}+\frac{\varepsilon}{q}=\frac{n}{2}+\frac{1}{4}+\frac{\varepsilon}{q}
$$

and combining (5) with (17) and (18) we obtain

$$
c^{n / 2+1 / 4+\varepsilon / q} \leq C c^{n / 2+1 / 4} .
$$

Taking $c$ large we conclude that $\varepsilon \leq 0$, which gives a contradiction. The proof of Theorem 2 is complete.

We finally remark that the method which we used in the proof of Theorem 2 to show that (5) cannot be improved, can also be used to prove that the $L^{4}$ estimate in (2) cannot be replaced by an $L^{q}$ estimate for $q>4$.

\section{References}

[1] J. Bourgain, 'A remark on Schrödinger operators', Israel J. Math. 77 (1992), 1-16.

[2] A. Carbery, 'Radial Fourier multipliers and associated maximal functions', in: Recent Progress in Fourier Analysis, North-Holland Mathematics Studies 111 (North-Holland, Amsterdam, 1985) pp. 49-56.

[3] L. Carleson, 'Some analytical problems related to statistical mechanics', in: Euclidean Harmonic Analysis, Lecture Notes in Math. 779 (Springer, Berlin, 1979) pp. 5-45.

[4] M. Cowling, 'Pointwise behaviour of solutions to Schrödinger equations', in: Harmonic Analysis, Lecture Notes in Math. 992 (Springer, Berlin, 1983) pp. 83-90.

[5] B. E. J. Dahlberg and C. E. Kenig, 'A note on the almost everywhere behaviour of solutions to the Schrödinger equation', in: Harmonic Analysis, Lecture Notes in Math. 908 (Springer, Berlin, 1982) pp. 205-209.

[6] C. E. Kenig and A. Ruiz, 'A strong type $(2,2)$ estimate for a maximal operator associated to the Schrödinger equation', Trans. Amer. Math Soc. 280 (1983), 239-246. 
[7] C. E. Kenig, G. Ponce and L. Vega, 'Oscillatory integrals and regularity of dispersive equations', Indiana Univ. Math. J. 40 (1991), 33-69.

[8] B. Muckenhoupt, 'Weighted norm inequalities for the Fourier transform', Trans. Amer. Math. Soc. 276 (1983), 729-742.

[9] E. Prestini, 'Radial functions and regularity of solutions to the Schrödinger equation', Monatsh. Math. 109 (1990), 135-143.

[10] P. Sjölin, 'Regularity of solutions to the Schrödinger equation', Duke Math. J. 55 (1987), 699-715.

[11] E. M. Stein and G. Weiss, Introduction to Fourier Analysis on Euclidean Spaces (Princeton Univ. Press, Princeton, 1971).

[12] L. Vega, 'Schrödinger equations: pointwise convergence to the initial data', Proc. Amer. Math. Soc. 102 (1988), 874-878.

Department of Mathematics

Royal Institute of Technology

S-10044 Stockholm

Sweden

email: pers@math.kth.se 